\title{
Maintainability Prediction of Object Oriented Software by using Adaptive Network based Fuzzy System Technique
}

\author{
Swati Mishra \\ SRIMT, Lucknow
}

\author{
Arun Sharma, $\mathrm{PhD}$ \\ IGDTUW, Delhi
}

\begin{abstract}
Today estimating the software size, cost and effort, effectively and accurately is probably the biggest challenge faced by software developers. It has major implications for the management of software development because both the over and under estimations have direct impact for causing damage to software companies. This process of changing the software which has been delivered is called software maintenance. The amount of resource, effort and time required and spent on software maintenance is much more than what is being spent on its development. The relationship between Object Oriented metrics and software maintenance effort is complex and non linear. Therefore, there is considerable research interest in development and application of sophisticated techniques which can be used to construct models for predicting software maintenance effort. The maintenance effort data of two commercial software products is used in the research. The dependent variable in this study is Change. The independent variables are five Object Oriented metrics out of nine proposed metrics on the basis of correlations between different metrics.
\end{abstract}

\section{General Terms}

Maintainability Prediction

\section{Keywords}

MI, Object Oriented Metrics (OO) metrics, UIMS, QUES, ANN, FIS, ANFIS, MATLAB ANFIS TOOLBOX.

\section{INTRODUCTION}

From the literature survey it can be concluded that Maintainability Prediction of OO software systems depends on many metrics. Rather we can say that different $\mathrm{OO}$ metrics work as independent variable for maintainability prediction [1]. Thus many researchers have taken different OO metrics into account and applied different techniques and models to predict maintainability. But since MI prediction share a complex relationship among them. Thus MI cannot be predicted by any irrelevant metrics with sufficient amount of accuracy. After neural network, Fuzzy approach for prediction was introduced and experiments were done by using various different networks like Fuzzy Inference System and Adaptive Network Based Fuzzy Inference Systems etc. various other approaches were also used for prediction using Fuzzy which were able to make prediction with good amount of accuracy. The technique was able to reduce the error up to a good level with different $\mathrm{OO}$ metrics. The proposed object oriented metrics helped to predict maintainability is given under table1 [2].
Table 1: METRIC DEFINITION TABLE

\begin{tabular}{|c|c|c|}
\hline $\begin{array}{l}\text { S. } \\
\text { NO. }\end{array}$ & Metrics & Definition \\
\hline 1 & WMC & $\begin{array}{l}\text { The sum of McCabe's cyclomatic } \\
\text { complexities of all local methods in a } \\
\text { class. Let a class K1 with method } \\
\text { M1...Mn that are defined in the class. Let } \\
\text { C1...... n be the complexity of the } \\
\text { methods. } \\
\qquad W M C=\sum_{i=1}^{n} C i\end{array}$ \\
\hline 2 & DIT & $\begin{array}{l}\text { The depth of a class in the inheritance } \\
\text { tree where the root class is zero. }\end{array}$ \\
\hline 3 & $\mathrm{NOC}$ & $\begin{array}{l}\text { The number of child classes for a class. It } \\
\text { counts number of immediate sub classes } \\
\text { of a class in hierarchy. }\end{array}$ \\
\hline 4 & RFC & $\begin{array}{l}\text { The number of local methods plus the } \\
\text { number of non local methods called by } \\
\text { local methods. }\end{array}$ \\
\hline 5 & LCOM & $\begin{array}{l}\text { The number of disjoint sets of local } \\
\text { methods. Each method in a disjoint set } \\
\text { shares at least one instance variable with } \\
\text { at least one member of the same set. }\end{array}$ \\
\hline 6 & MPC & $\begin{array}{l}\text { The number of messages sent out from a } \\
\text { class. }\end{array}$ \\
\hline 7 & DAC & $\begin{array}{l}\text { The number of instances of another class } \\
\text { declared within a class. }\end{array}$ \\
\hline 8 & NOM & The number of methods in a class. \\
\hline 9 & SIZE1 & $\begin{array}{l}\text { The number of lines of code excluding } \\
\text { comments. }\end{array}$ \\
\hline 10 & SIZE2 & $\begin{array}{l}\text { The total count of the number of data } \\
\text { attributes and the number of local } \\
\text { methods in a class. }\end{array}$ \\
\hline 11 & CHANGE & $\begin{array}{l}\text { The number of lines added and } \\
\text { deleted in a class, change of the } \\
\text { content is counted as two. }\end{array}$ \\
\hline
\end{tabular}




\section{LITERATURE SURVEY}

Li and Henry have given their research on object oriented metrics which predict maintainability. Their aim was to propose \& give implementation for the object oriented metrics and make investigation for their co-relation with the maintenance effort. They have validated the model on two object oriented systems UIMS and QUES. These are two datasets available for software maintainability prediction.

The study of Shing and Jang [3] in 1993 was based on ANFIS in place of conventional mathematical tools. He has implemented the framework of adaptive networks which can build an input- output mapping on different data pairs. In his study he also compared the proposed model for predicting chaotic time series with artificial neural network and fuzzy modeling and he found ANFIS perform outstanding as much as we provided sufficient amount of training data.

Dr. Sallie Henry [4] in his paper in 1993 discussed and analyzed three studies on $\mathrm{OO}$ metrics and also given the study on reusability through these metrics. He included the thought that object oriented Paradigms over procedural paradigms and claimed to aid in software reuse and software maintainability.

K. K. Aggarwal [5] discussed in their publication in 2006 on artificial neural network used for maintainability prediction through object oriented metrics. They discussed the rapid application of ANN used in providing the model for predicting quality attributes for $\mathrm{OO}$ systems. In their study they included quality estimation i.e. maintainability of software by the dependent variable as maintenance effort and independent variable as eight $\mathrm{OO}$ metrics.

Riaz et. al [6] in 2009 in their paper has given a systematic review on software maintainability prediction and metrics for OO systems. They have collected 710 studies and shortlisted 15 to validate and provide evidence to their study. They made this review on the basis of a set of meaningful review questions.

Dr. Kaur et. al [7] in 2010 in their international journal discussed the relationship between object oriented metrics and software maintenance effort i.e. complex and non linear. In this paper they have evaluated and compared different application of soft computing techniques, for predicting software maintenance effort. They used data of two commercial software products and observed that soft computing techniques can be used for constructing more accurate models for predicting maintenance effort of $\mathrm{OO}$ systems. They compared the ANN, Fuzzy Inference system and adaptive network based fuzzy inference systems and found adaptive network based fuzzy inference system most accurate for prediction for the applied dataset.

Mr. Sandeep Sharawat [8] in 2012 has focused on increasing the maintainability index value for better maintenance prediction of software systems, which is a composite metric. He used Li- Henry data for prediction of MI to train neural network and tested the data for different neural network algorithms available in matlab neural network toolbox and found that Trainlm training function is most suitable for prediction on given dataset. He concluded that his work can be used to predict MI in the industries for software application development in such a way that the application must be more maintainable and can be used in risk or cost analysis, which can help to reduce overall cost of software systems.
Gagan Tiwari and Arun Sharma [9] in their paper in 2012 have given a systematic survey on different maintainability techniques for software development. In their research they have given a detailed survey on the work of various researchers work for predicting better maintainability so that time, effort and money involved in maintaining a software can be utilized in effective manner as its always a major concern than the software development time.

Roheet Bhatnagar and Mrinal Kanti Ghose [10] in 2012 in their paper focused on the software size, cost, effort and schedule estimation which is a biggest challenge in SDLC phases. They included ANN and Fuzzy Logic i.e. two most popular soft computing technique for their study on effort estimation. They have given detailed discussion on predicting maintenance effort at early stages of software development.

In their paper in 2012, Neha et. al [11] discussed the fuzzy layered approach of maintainability of object Oriented Software System. They worked on a comprehensive model to evaluate the maintainability of Object Oriented Software System based on fuzzy layered approach. They also found that maintainability evaluation is an essential component of modern software development life cycle. Their study developed a qualitative evaluation of OO software Systems based on Fuzzy approach and identified certain design and code characteristic.

Saini and Chauhan [12] in their paper in 2013, has given a roadmap of all the existing models used for maintain a software either it is an object oriented software or web design system for rapidly changing environment and requirement changes. They have also collected data from industry, research centers and student projects. They have given a brief view on the modules or metrics required for software maintainability. They have taken design matrices as well as source code matrices into consideration for their work.

\section{EMPERICAL DATA}

For maintainability prediction first of all the data is selected, for which the change variable is to be predicted. If we want to predict the change in UIMS dataset we will take into consideration QUES. This data will be considered as the training data. This data is taken into account with the fact that the OO metric data observed for the UIMS system can be trained by QUES data. Thus we must have sufficient information about the OO metric of QUES system [13].

The size of the data to be analyzed is 71 . This value is in agreement with the size of data requirement for our study. We have normalized this data to get more accurate result and to predict the values within range. The input size is 5 metric where output is a single metric value. This provides a normalized view thus eliminates the problem of over-fitting the data while training the network.

We used following formula to normalize the data:

$$
V^{\prime}=\left(\max _{T}-\min _{T}\right)\left[\frac{V-\min _{A}}{\max _{A}-\min _{A}}\right]+\min _{T}
$$

$\max _{\mathrm{T}}=$ maximum desired target value i.e. 1 $\min _{\mathrm{T}}=$ minimum desired target value i.e. 0 $\max _{\mathrm{A}}=$ maximum range of actual data $\min _{\mathrm{A}}=$ minimum range of actual data 


\section{EXPERIMENTAL RESULT}

\subsection{Simulation}

One of the characteristic of adaptive network based fuzzy networks is that they are not programmed rather, they are trained [14]. The ANFIS Editor GUI window includes four distinct areas to support a typical workflow. The GUI lets one perform the following tasks:

- $\quad$ Loading, Plotting, and Clearing the Data

- Generating or Loading the Initial FIS Structure

- $\quad$ Training the FIS

- $\quad$ Validating the Trained FIS

Thus we will be training the network with the help of QUES data for the same five input metrics and with the normalized values. Certain training inputs are provided to the network using which they generalize and adapt the trends. There after they are able to use their knowledge in giving the output when certain inputs are given. Thus the data after pre-processing is used for simulating the network.

\subsection{Train the Network}

Network model is trained with the data of QUES system for which prediction in the UIMS system is to be done. To this, specifications are added by training it with the five metric i.e. DIT, NOC, MPC, RFC and SIZE2. The input size we used is 5 i.e. the five $\mathrm{OO}$ metrics which are used for predicting the change variable on which maintainability of $\mathrm{OO}$ software depends.

$\begin{array}{ll}\text { Therefore, } & \text { Training Set: } 71 \\ & \text { Testing Set: } 39\end{array}$

Thus we will be training the network using 71 data values of QUES System after which 39 values of UIMS system will be used to test the network.

\subsection{Prediction}

Once the training of the network is done it is provided with the testing data. Using this input data used as training data now the network predicts the output. We now compare the trained value trends with the testing output trends to calculate the error in prediction. This process is done for all the data available and at the end, the average error is calculated to analyse the efficiency of the network.

\subsection{Working Algorithm}

We will be using the following algorithm:

- Analyse the correlation between the ten Object Oriented metrics.

- On the basis of correlation we reduced the ten metrics to five in our work.

- Perform normalization of data

- Simulate the model using the model generated using ANFIS editor: Fuzzy Logic toolbox in MATLAB.

- Train the network using the QUES training data.

- Generate FIS for training data

- Now train the network for training data

- Load the test data

- Generate FIS for test data on the basis of training data

- The network will now generate the future value as output.

- Test the network

- Compare it with the already present data and calculate the average testing error.

\subsection{Experimental Results}

The maintainability prediction on the basis of change variable of OO software used UIMS data for verifying the results obtained from the model generated in the study. 39 test values are observed and test to find the result. The detailed results achieved are shown below. The tables have the following attributes:

- DIT: Depth of Inheritance Tree.

- NOC: Number of Children

- MPC: Message Passing Coupling

- RFC: Response for a Class

- SIZE2: number of attribute + number of local methods

- CHANGE: variable for predicting maintainability

After this, an Adaptive network based fuzzy approach came which used one factor for predicting the results that was able to make considerable improvements in the results. But we implemented the technique and reduced $\mathrm{OO}$ metrics to five. We have taken the data of UIMS and QUES to prove the technique and we have shown the results in the above mentioned tables after calculating the errors. This technique reduced the average error rate up to $21.711 \%$ considerably improves the results.

Fig 1: FIS Output and Average Calculated Error Diagram

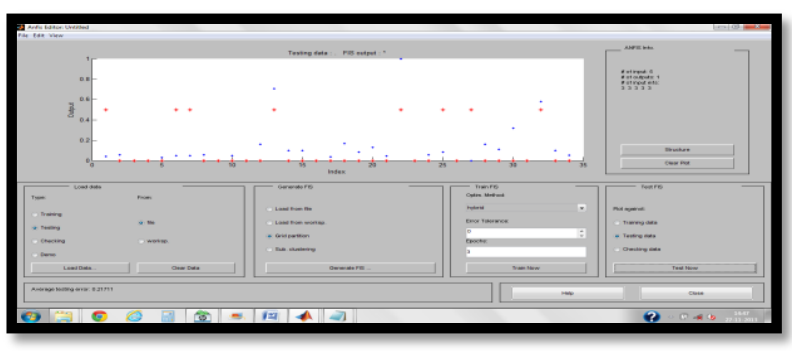

\section{CONCLUSIONS}

This thesis aims of implementing and enhancing the existing technique of maintainability effort by reducing the ten $\mathrm{OO}$ metrics to five and hence getting the results. As we know that maintainability prediction is nowadays an important topic for research. This work studied the various soft computing techniques to implement software maintainability and implemented the result by Adaptive Neuro Fuzzy technique which uses QUES training data for predicting the value of change variable.

On the basis of detailed literature survey we have taken the papers from the year 1993 to 2013. Based on which we were able to derive the motivation for the dissertation. From the survey it is clear that using ten $\mathrm{OO}$ metrics increasing the overhead of the working mechanism so if we can reduce it to some extent it will be helpful for implementation. We have achieved $21.711 \%$ accuracy with the reduced number of metrics. Also from the literature survey it was concluded that many techniques were used for prediction but they lacked in accuracy but comparatively Adaptive Neuro Fuzzy based technique give most effective results. The Adaptive Neuro based Fuzzy interface is designed in MATLAB using ANFIS Tool kit. Also the network is simulated trained and tested through the same tool and different data sets.

From the experimental results we reach to the conclusion that the five $\mathrm{OO}$ metric based maintainability predictions gives better result. This technique was able to predict the maintainability in terms of change variable by using five 
metrics in place of ten metrics which reduce the overhead of calculating the same output with ten OO metrics.

\section{FUTURE SCOPE}

As the future scope is concerned the work finds its application in software engineering domain which shows object oriented behaviour due to the cumulative effect of various contributing $\mathrm{OO}$ metrics. This interesting behaviour shown by them is due to the merger of their individual effects and correlation of ten metrics. These correlations show the nature and behaviour of OO metrics with another, but their nature and dependency will serve as model for any other software prediction accurately. This in turn increases the probability of exploring the trends in near future. Moreover,

- A much better generalized approach is expected if we consider the data over past many years, trained the network for more large data.

- Also we could even reduce more metrics and can see the cumulative effect of those metrics on the future prediction.

- We can also give a comparative study with other soft computing techniques.

\section{REFERENCES}

[1] "Extension of Object Oriented Metrics Suite for Software Maintenance" available at http://www.hindawi.com/isrn/software.engineering/2013/ 276105/

[2] Ruchika Malhotra and Anuradha Chug, - Software Maintainability Prediction using Machine Learning Algorithms\|, International Journal (SEIJ), Vol 2, No. 2, September 2012

[3] Jyh Shing, Roger Jang, -ANFIS: Adaptive Network based Fuzzy Inference Systemll, IEEE Transactions on Systems, Man and Cybernetics, vol. 23 ,no 3, pp 665685,1993 .

[4] Dr. Sallie Henry, "Measurement of Software Maintainability and Reusability in the Object Oriented Paradigm", Virginia Tech, Blacksburg, Virginia, September 6, 1994.

[5] K. K. Aggarwal, Yogesh Singh, Arvinder Kaur and Ruchika Malhotra, "Application of Artificial Neural Network for predicting Maintainability Using Object
Oriented Metrics", World Academy of Science, Engineering and Technology 222006.

[6] Mehwish Riaz, Emilia Mends, Ewan Tempero, "A Systematic Review of Software Maintainability Prediction and Metrics", Third International Symposium on Empirical Software Engineering and Measurement,978-1-4244-4841-8/09/\$25.00 (C) 2009 IEEE.

[7] Dr. Arvinder Kaur, Kamaldeep Kaur and Dr. Ruchika Malhotra, "Soft Computing Approaches for Prediction of Software Maintenance Effort", 2010 International Journal of Computer Applications(0975-8887), Volume1-No.16

[8] Mr. Sandeep Sharawat,"Software Maintainability Prediction Using Neiral Networks", International Journal of Engineering Research and Applications (IJERA), Volume 2, Issue 2, Mar- APR 2012, PP.750-755

[9] Gagan Tiwari and Arun Sharma, "Maintainability Techniques for Software Development Approaches- A Systematic Survey", Special Issue of International Journal of Computer Applications (0975-8887), ICNICT 2012, November 2012.

[10] Roheet Bhatnagar and Mrinal Kanti Ghose, Comparing Soft Computing Techniques For Early Stage Software Development Effort Estimationsll, International Journal of Software Engineering \& Applications (IJSEA), Vol. 3, No. 2, March 2012.

[11] Neha Goel, Sanjay Kumar Dubey and Prof. (Dr.) Ajay Rana, "Fuzzy Layered Approach for Maintainability Evaluation of Object Oriented Software System", International Journal of Scientific \& Engineering Research, Volume 3, Issue 6, June-2012.

[12] Monika Saini and Mukti Cahuhan, "A Roadmap of Software System Maintainability Models", Journal of Software \& Web Sciences (IJSWS) 12-359; (c) 2013, IJSWS All Rights Reserved.

[13] Wei Li and Sallie Henry, "Object Oriented Metrics Which Predict Maintainability", Department Of Computer Science, Virginia Polytechnic Institute and State University, Blacksburg, Virginia, February 1, 1993.

[14] MATLAB/ANFIS editor: Fuzzy logic toolbox. www.mathworks.in. 